\title{
Rutas del aguardiente en el Cono Sur de América (siglos XVI-XIX). Antecedentes de la Denominación de Origen Pisco
}

\author{
Routes of brandy in Southern Cone of America (1586-1850). \\ Background of Appellation of Origin Pisco
}

\author{
Pablo Lacoste Gargantini ${ }^{*}$, Diego Jiménez Cabrera ${ }^{2}$, Enrique Cruz ${ }^{3}$, Bibiana Rendón Zapata ${ }^{4}$, \\ Natalia Soto González ${ }^{5}$, Mario Solar ${ }^{5}$, Carolina Polanco ${ }^{6}$
}

\section{RESUMEN}

Entre los siglos XVII y XIX el Norte Chico de Chile se convirtió en un dinámico polo de producción y exportación de aguardientes. El aguardiente se destinaba al mercado de Potosí, tanto por vía marítima como por vía terrestre, gracias al servicio regular de transporte de carga que ofrecían los arrieros. Surgió así la ruta del aguardiente, que contribuyó a establecer estrechos lazos económicos, sociales y culturales entre las ciudades, villas y localidades rurales de las actuales Chile, Argentina y Bolivia. Así se echaron las bases para el desarrollo de la Denominación de Origen Pisco, delimitada en 1931.

Palabras clave: aguardiente, rutas comerciales, vino, pisco, denominaciones de origen, industria vitivinícola.

\section{ABSTRACT}

Between the seventeenth and nineteenth centuries, the North of Chile became a dynamic center of production and export of wine brandy called "pisco". The spirits market was intended to Potosi, both by sea and by land, by regular freight service offered by the carriers. Thus arose the path of brandy, which helped to establish close economic, social and cultural links between the cities, towns and rural areas of current Chile, Argentina and Bolivia. So the basis for the development of the Appellation of Origin Pisco bounded burst in 1931.

Key words: grape brandy, pisco, appellations of origin, wine industry.

Dentro de la industria vitivinícola, el aguardiente constituye una rama significativa. En el norte de Portugal surgieron los vinos y aguardientes de Oporto (Bennett, 2005); en el sur de España se consolidó el brandy de Jerez (De las Cuevas, 2003). En Francia se hicieron notables los aguardientes de Cognac y Armagnac. En América Latina el producto emblemático es el pisco, tanto en Perú (Huertas, 2012; Rice, 2012) como en Chile (Cortés, 2005; Lacoste et al., 2013 y 2014).

Debido a su alta graduación alcohólica, el aguardiente ha cumplido importantes funciones medicinales como desinfectante para las heridas, como analgésico y para otras funciones. Muy apreciado por distintas culturas, el aguardiente fue llamado con frecuencia "el agua de la vida", eau de la vie. También sirvió para impulsar la vitivinicultura, con la producción de vinos generosos y el fortalecimiento de vinos débiles (De las Cuevas, 2003; Maldonado, 1999). El alambique impulsó avances en distintos campos, desde la formación de los primeros químicos farmacéuticos y alquimistas hasta el descubrimiento de la destilación alcohólica, que se estima fue lograda por primera vez entre los siglos XII y XIII (Forbes, 1948, 55-98). Gracias al alambique, a fines de la Edad Media se comenzaron a obtener los primeros destilados (Forbes, 1948; Maldonado, 1999; De las Cuevas, 2003; Marchena, 2010). Al principio fueron producciones acotadas y limitadas, pero de esta manera de echaron las bases para la expansión del alcohol

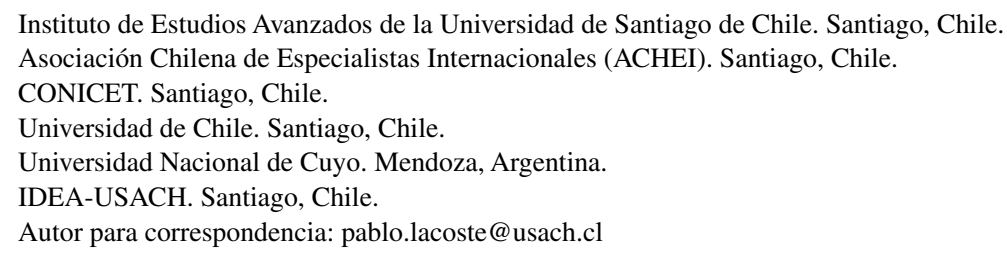


y el aguardiente, proceso que se consolidó entre los siglos XVII y XVIII (Braudel, 1994).

Los estudios específicos sobre el aguardiente de uva en América del Sur han permitido conocer la relevancia que esta industria alcanzó tempranamente en las zonas vitivinícolas de la región, sobre todo en Perú (Huertas, 2012; Rice, 2012). En cambio para el caso chileno los avances han sido mucho más modestos (Cortés, 2005; Lacoste et al., 2013). La mayor producción historiográfica sobre el pisco peruano sobre el pisco chileno han creado una imagen distorsionada sobre el desarrollo de ambas industrias. Sin embargo, ya se han detectado algunos indicadores relevantes.

La producción de vinos y aguardientes en el corregimiento de Coquimbo comenzó en el siglo XVI, con la conquista española. A partir de entonces los valles del Elqui, Limarí y otros pusieron en marcha el proceso de cultivar las viñas, elaborar el vino y destilar aguardientes. Hacia 1790 el corregimiento de Coquimbo elaboraba 40.000 arrobas de vino y 5.000 arrobas de aguardiente, destinado a abastecer los mercados mineros internos y la demanda de Potosí (Pinto, 1980). El principal mercado regional era sin dudas Potosí, sobre todo para el aguardiente. En 1733 el aguardiente representó el 21,4\% del total de los productos de la tierra importados por Potosí, lo que representaba el segundo rubro más relevante, solo superado por los textiles; pero el tráfico de aguardiente fue en ascenso, hasta llegar al $30,6 \%$ en 1793, quedando entonces en el primer lugar. En ese momento el valor del aguardiente importado llegó a \$258.954 (Tándeter, 1987). Entre los proveedores de aguardiente para Potosí figuraban en primer lugar las regiones productivas de Perú. De todos modos, la exportación de aguardientes chilenos a Potosí llegó a generar una competencia importante a los productores peruanos. El flujo de aguardiente del norte chico de Chile fue en contante aumento, hasta afectar los intereses de las haciendas peruanas. Como resultado, los productores de esta región resolvieron apelar a las autoridades españoles para solicitar privilegios de acceso al mercado de Potosí. Para avanzar en esta dirección, tomaron la decisión de solicitar medidas directas del Consejo de Indias: "Los hacendados vinícolas de Moquegua pidieron con grande insistencia al Consejo de Indias que de ningún modo se permitieran las plantaciones de viñas en La Paz (Alto Perú) y que se cortara a todo trance la introducción en Potosí de los aguardientes de Chile" (Assadourian, 1983).
Se produjo una auténtica guerra comercial entre el aguardiente peruano y el aguardiente chileno por el control del mercado de Potosí en el periodo colonial. La menor distancia física entre la zona de producción y el mercado de consumo favorecía a los hacendados peruanos, igual que la cercanía del virrey, en Lima, y la mayor capacidad de influencia en la corte. De todos modos, los productores chilenos lograron hacer frente a estas maniobras con la ayuda que representaba la ventaja tecnológica de los alambiques de Coquimbo sobre las falcas peruanas, y el eficaz servicio regular de transporte terrestre que aseguraban las tropas de arrieros.

La destilación alcohólica generó una serie de encadenamientos productivos que facilitaron el sostenido desarrollo de la minería del Cerro Rico de Potosí (Rice, 2012). La industria vitivinícola generó mercados de las fábricas de botijas, botellas de vidrio, odres de cuero y cubas, entre otros productos que dieron lugar a una dinámica economía colonial (Romano, 2004). Estos últimos constituyeron encadenamientos productivos, que a la postre se convirtieron en verdaderos encadenamientos culturales, que forjaron la configuración de un espacio social colonial, tanto en el Virreinato del Perú (Huertas, 2004 y 2012) como en el Cono Sur de América en su conjunto, que forjaron un verdadero hermanamiento ancestral, proceso similar al que se produjo en las antiguas rutas del vino en Europa (Fernández, 2005).

Durante su auge en los siglos XVI y XVII el aguardiente fue uno de los pilares de la industria colonial en Chile y Perú, adquiriendo un considerable nivel de concentración productiva dentro de este último (Romano, 2004, 248-249). El aguardiente sirvió de base material para el desarrollo de una economía-mundo. El alto valor de este producto facilitaba el surgimiento de largas rutas comerciales y densas redes económicas y sociales. En ese sentido, es relevante conocer estos procesos que, a pesar de mantenerse invisibles durante largos siglos, alcanzaron mayor visibilidad con la delimitación de la DO Pisco en 1931 (Lacoste et al., 2014a). Se trata, por lo tanto, de conocer la etapa anterior.

Hasta el momento poco se ha podido aclarar sobre algunos aspectos específicos, particularmente las rutas meridionales del aguardiente. La historiografía peruana ha señalado las rutas desde las zonas productivas peruanas hacia los mercados de Potosí (Huertas, 2012), mientras que menor atención han merecido las rutas que llegaban a ese centro minero 


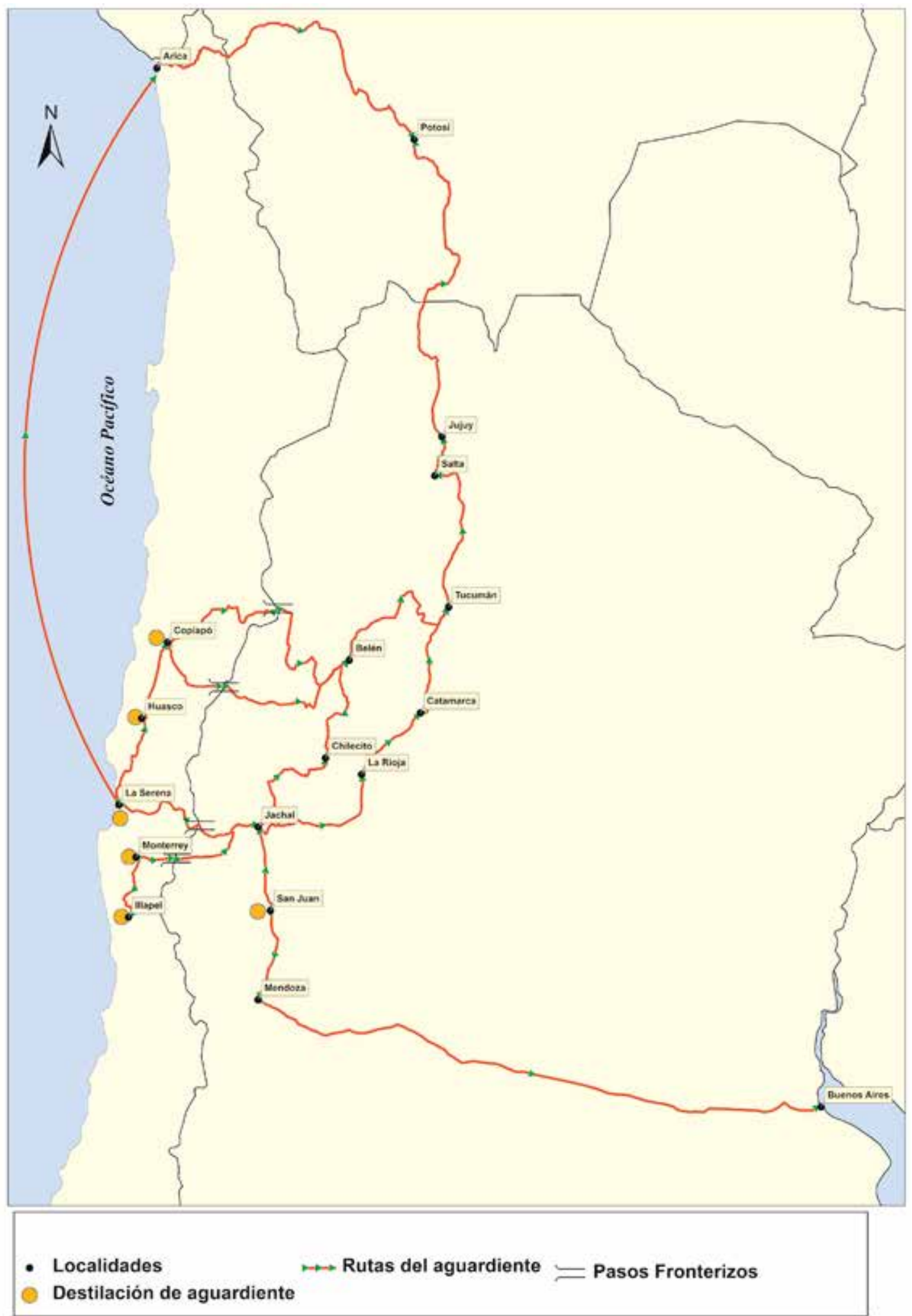

Bibiana Rendón, Proyecto Fondecyt 1130096

Figura 1. Rutas del aguardiente de uva, siglos XVII-XVIII-XIX. 
desde el sur. Corresponde entonces avanzar en el estudio de las rutas de la zona sur del área productiva en la región.

\section{La ruta del aguardiente: el triángulo La Serena - Potosí - Buenos Aires}

El surgimiento de Potosí como el gran centro minero de la región configuró un mercado atractivo para los productores de toda la región. Basta recordar que la prosperidad de la producción de plata en Potosí atrajo población hasta alcanzar niveles sin precedentes. Desde su descubrimiento en 1545 hasta el final de la Colonia, en 1825, Potosí se desempeñó como un emporio de producción minera y actividad económica. En 1611 la población de Potosí llegó a 150.000 habitantes, cuando Santiago de Chile apenas contaba con 10.000. La supremacía de Potosí como principal mercado de la región se mantuvo durante todo el periodo colonial. Basta recordar que en 1778 la población llegaba a 40.000 habitantes en Santiago de Chile; la gobernación de Tucumán tenía 85.000; Córdoba 40.000, Buenos Aires 37.000 y Cuyo 23.000 (dentro de la provincia de Cuyo las principales ciudades eran San Juan (7.690 almas en 1777) y Mendoza (8.765 en 1777 según Martin de Moussy)). Particular interés para los productores y exportadores de Coquimbo representaba la gobernación de Tucumán por contar con seis ciudades de entre 10 y 20 mil habitantes. En 1778 la gobernación del Tucumán representaba el $40 \%$ de la población rioplatense. De acuerdo con el Padrón de 1778, la población era la siguiente: Córdoba: 40.022 habitantes; Buenos Aires: 37.130; Tucumán: 20.104; Santiago del Estero: 15.456; Catamarca: 15.315; Jujuy: 13.619; Salta: 11.565; La Rioja: 9.723 (Bazán, 1995).

Las correlaciones de población permiten comprender el significado de los mercados de Potosí y el noroeste argentino para la economía del Norte Chico de Chile. Este tenía una ciudad (La Serena) y cuatro villas: Copiapó, Vallenar, Combarbalá e Illapel. A ello se sumaban algunas haciendas que generaban movimiento y animación, sentando las bases de futuras localidades, como Monterrey (actual Montepatria) y Marquesa Alta (actual Vicuña), y la población rural dispersa en pequeños caseríos. $\mathrm{La}$ población total del Norte Chico creció de 12.000 habitantes en 1700 a 17.000 en 1744 y 34.000 en 1778 (Pinto, 1992). En ese momento, su única ciudad, La Serena, apenas contaba con 2.000 habitantes.
La simple observación de estas cifras de población permite comprender el significado que tenían los mercados del NOA y Potosí para los productores del Corregimiento de Coquimbo. Es fácil captar el significado que tenían los 150.000 habitantes de Potosí y los 85.000 de la gobernación de Tucumán para los productores de una ciudad como La Serena, que apenas tenía 2.000. Este interés fue suficiente para alentar a los arrieros a cruzar la cordillera de los Andes, para ello era necesario ascender hasta más de 4.500 metros de altura snm para atravesar los puertos y recién entonces descender por la falda oriental de la montaña. El derrotero era exigente y requería mucho esfuerzo, pero pronto aparecía la compensación, al encontrarse el ramillete de ciudades de 10.000 habitantes o más, lo que representaba también un mercado atractivo y una base eficaz de abastecimiento de los arrieros a lo largo del viaje. Esta red de villas y ciudades facilitó el transporte de vinos, aguardientes, frutas secas, cobre labrado y demás productos desde el Norte Chico hasta Potosí (Lacoste et al., 2014a).

Las rutas marítimas utilizaron el puerto de Arica como portal de ingreso. Hasta allí llegaban los barcos con productos chilenos, provenientes de los puertos de Talcahuano, Valparaíso y Coquimbo. De este último provenían los principales cargamentos de aguardiente. Una vez en Arica, las cargas se trasbordaban a las recuas de mulas, y los arrieros las llevaban hacia Potosí. Estos flujos comerciales tendieron también a promover contactos con los vecinos de Alto Perú que llegaban al NOA, Cuyo y Norte Chico, a promover sus negocios. Así, por ejemplo, en 1748 testificó en un informe matrimonial, celebrado en Mendoza, Hilario de los Ríos, natural de Chuquisaca, el que relató detalles de sus viajes por Potosí y distintas ciudades de la gobernación de Tucumán (declaración de Hilario de los Ríos en el Informe Matrimonial del enlace entre doña Tomasa Ponce de León y José de Arellano, Mendoza, 8 de marzo de 1748. Archivo del Arzobispado de Mendoza, Parroquia Matriz de Mendoza, Libro de Informaciones Matrimoniales, año 1747-1748, foja 128.)

Las rutas terrestres del pisco partían en los centros de producción del Norte Chico chileno. Como se ha señalado en otra parte (ver Idesia 32), en el valle del Elqui se levantó la primera pisquera de América en el primer tercio del siglo XVIII y a partir de entonces se comenzó a usar el nombre pisco para denominarlo. Los arrieros cargaban aguardientes 


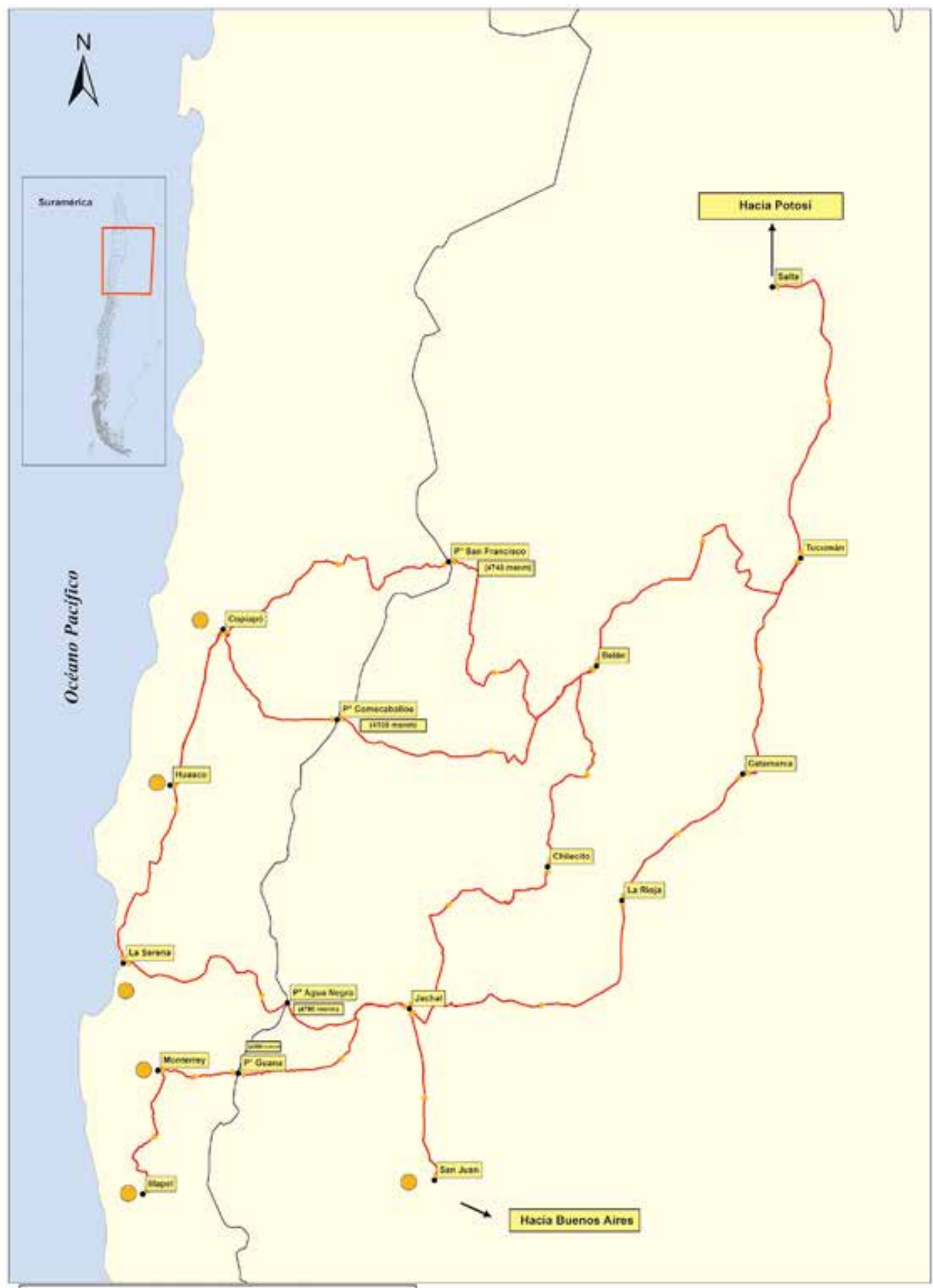

- Localidades

- Destilación de aguardiente

_._Ruta del aguardiente

Bibiana Rondón, Proyecto Fondecyt 1130096

Figura 2. Rutas del aguardiente de uva en Chile y Argentina (detalle), siglos XVII-XVIII-XIX. 
en el valle del Limarí y el valle del Elqui, cruzaban los Andes por pasos como Guana y otros, y se encontraban en Jáchal; allí llegaban también los arrieros que venían desde San Juan, y juntos seguían rumbo al norte. Algunos tomaban el camino hacia La Rioja, Catamarca y Tucumán. Otros andaban la ruta de Chilecito y Belén. Allí se encontraban con los arrieros que venían de los valles del Huasco y Copiapó y habían cruzado la cordillera por los pasos San Francisco y Comecaballos, respectivamente. Así se consolidó Belén como punto de encuentro de arrieros de distintas ciudades de Chile. Desde allí continuaban el camino hasta Tucumán. Hasta ese lugar el camino era de herradura. En Tucumán comenzaba el camino carretero principal, llamado "Carrera del Norte", que enlazaba Buenos Aires con Salta y Jujuy. Luego el camino volvía a ser de herradura, para continuar rumbo a Potosí por la quebrada de Humahuaca (Lacoste et al., 2014b).

En el periodo colonial el flujo de aguardiente desde Chile hacia Potosí no alcanzó las dimensiones del aguardiente de origen peruano. Este mantuvo su liderazgo durante el siglo XVII y buena parte del XVIII. En 1794 Potosí importó de Moquegua $\$ 100.000$ en vino (10.000 botijas) y $\$ 1.000 .000$ en aguardiente (50.000 cuartillos). De todos modos el aporte chileno alcanzó una porción de mercado lo suficientemente considerable como para despertar la preocupación de los productores peruanos, los cuales realizaron gestiones ante el Consejo de Indias con vistas a lograr que se prohibiera el comercio de aguardiente de procedencia chilena (Assadourian, 1983).

Los productores de aguardiente chileno dedicados a esta actividad pertenecían a distintos estratos, incluyendo grandes y pequeños empresarios. Entre estos últimos cabe señalar, por ejemplo, el caso de Jerónimo Toro. Poseía un modesto establecimiento vitivinícola, con sus viñas, tinajas, un fondo de cobre de cinco arrobas de peso, una piquera de tres arrobas y un alambique. A pesar de disponer solo de modestas instalaciones, se sumó al tráfico de Potosí; estableció lazos de confianza con comerciantes locales, colocó productos, e integró una red de confianza y crédito. Al redactar su testamento en Santiago (1726), junto con la enumeración de sus bienes, se refirió a los lazos que tenía en Potosí, y reconoció que tenía deudas con varios vecinos de esa localidad; debido a sus escasos medios, solicitó que aquellos le perdonaran sus deudas (testamento de Jerónimo Toro, Santiago de Chile, 8 de setiembre de 1726. AN, FES, Pieza 68, Foja 168). La figura de Jerónimo Toro representa al conjunto de pequeños productores chilenos que se animaron a comerciar con Potosí.

Para asegurar la fluidez de los circuitos comerciales se construyeron redes de contactos entre los lugares de producción y los de consumo. Vecinos de cada uno de estos dos lugares viajaban al otro para realizar negocios. En algunos casos las familias se dividieron, para asentar algunos de sus miembros en Potosí y otros en Chile, de modo tal de asegurar el funcionamiento de las redes comerciales. Así, por ejemplo, el productor y comerciante de San Juan, Jacinto Echegaray, señaló que "conduje a mi mujer a la Villa de Potosí donde al presente se haya con mi familia" (testamento de Jacinto Echegaray, San Juan, 7 de mayo de 1777. Archivo del Poder Judicial de San Juan, Protocolo 1777-1778, foja 84).

La estrategia era encargar los negocios a una parte de la familia, que viajaba a Potosí, mientras la otra permanecía en Chile cultivando la viña, elaborando el vino y destilando el aguardiente. Doña Magdalena Garramuño viajaba al Alto Perú, y dejaba a sus hijos en las tareas vitivinícolas en Chile: "mi hijo don Bailón hizo durante la ausencia mía en Potosí la cosecha de la viña en cuatro años consecutivos" (testamento de Magdalena Garramuño, junio de 1798. APJSJ, Protocolo 1798, folio 88-88).

La ruta del aguardiente que fluía de Chile hacia Potosí era un camino de ida y vuelta. Por lo general, el aguardiente se pagaba con la abundante plata que se producía en Potosí. Luego ese dinero se reinvertía en otros productos comprados en otras ciudades del norte (como Lima), y se regresaba con esas mercaderías para venderlas en Chile. En ese sentido, fue interesante la producción textil. Los inventarios de bienes chilenos han registrado productos originarios de Potosí, sobre todo lienzos y sombreros.

Potosí fue el mercado principal para el pisco aguardiente chileno, pero no el único. Este producto también se distribuyó en las provincias del Río de la Plata, como Tucumán, Córdoba, Santa Fe, y sobre todo Buenos Aires. Cientos de pulperías florecieron tanto en la ciudad como en la campaña y allí se aseguraba la distribución y consumo de aguardientes de Cuyo, que formó parte del Reino de Chile desde 1561 hasta 1778 (Mayo, 2000; Carrera, 2011).

La ruta del aguardiente de Cuyo a Buenos Aires tenía una ventaja importante dada por las 
planicies, que facilitaban el transporte en carretas. En el segundo tercio del siglo XVIII los productores cuyanos remitían 8.000 botijas por año a Buenos Aires, donde se vendían a poco más de $\$ 11$ por botija. Este lucrativo comercio despertó el interés de las autoridades para incrementar los ingresos fiscales. Para ello, el gobernador de Córdoba del Tucumán estableció un impuesto de $\$ 6$ por botija de aguardiente en el fuerte de Río IV, lo que generó una serie de conflictos con la industria. El impuesto puso en peligro la viabilidad de la industria y para eludirlo algunos troperos optaron por eludir el control oficial, tomando rutas por el sur, ello implicaba adentrarse en los territorios controlados por los pueblos indígenas; surgieron choques armados y varios troperos murieron a manos de los indios, lo que generó serios escándalos que fueron abordados por el virrey del Perú, tal como se ha estudiado en otra parte (Lacoste, 2005).

Hacia fines del siglo XVIII se consolidó el circuito regional del aguardiente con sus dos rutas principales, la del norte rumbo a Potosí, y la del este, con destino a Buenos Aires. Cada una tenía sus propias características: el aguardiente del norte se transportaba en mulas, envasada en odres de cuero de chivato; en cambio la ruta del este se recorría en carretas con barriles de madera o botijas de greda. Los productores de aguardiente debían ampliar sus redes para abarcar estos distantes mercados. Un símbolo de este movimiento lo representó, por ejemplo, don Dionisio Jofré, productor de aguardiente asentado en San Juan; poseía una tropa de 80 mulas de carga, y despachaba sus alcoholes tanto hacia Tupiza (Potosí) como a Buenos Aires (testamento de Dionisio Jofré, San Juan, 3 de abril de 1787. APJSJ, Protocolo 1787-1788, Fojas 32-43).

La calidad del aguardiente tenía algunas variaciones según la región. Por un lado se distinguía el aguardiente de vino del aguardiente de orujo. El aguardiente de vino era el más cotizado y podía valuarse en \$ 10 por arroba. En La Serena se registraban estas operaciones, y a veces también en localidades ubicadas más al sur. De todos modos, en el Valle Central de Chile se difundió ampliamente el aguardiente de orujo, sobre todo en los corregimientos de Quillota, San Fernando y Cauquenes. En San Juan, Santiago y San Felipe se elaboraba aguardiente anisado. En Mendoza se registraron casos de aguardiente añejo y aguardiente a la vela, proceso parecido al que se empleaba en el brandy de Jerez. También se difundió aguardiente de doble destilación, llamado "aguardiente resacado". Esta modalidad se difundió ampliamente en San Juan y, en menor medida, en el Valle Central de Chile. El aguardiente resacado tenía mayor trabajo y graduación alcohólica, motivo por el cual tenía mejor precio. En San Fernando se registró una partida de aguardiente de mala calidad que no se tasó por no estar resacado. Más allá de estos matices, lo importante es destacar que ya en el siglo XVIII se fue trazando una diferencia del tipo de aguardiente: en la zona sur, tanto en Mendoza como en el Valle Central de Chile, la tendencia principal era destilar aguardiente de orujo, a la vez que el mosto se dedicaba a elaborar y comercializar vino; en cambio más al norte, en San Juan y en el corregimiento de Coquimbo, hubo una tendencia a elaborar más aguardiente de vino.

\section{Conclusión}

En el periodo colonial se produjo el surgimiento y consolidación de la producción de aguardientes en Chile, proceso facilitado por la demanda de los mercados -sobre todo Potosí- y las ventajas comparativas que representaban la disponibilidad de cobre, la cultura del cobre labrado, la abundancia de viñas y vinos en la región. A ello se sumó la presencia de las villas y ciudades del noroeste argentino que facilitaron la transitabilidad de los caminos a los arrieros chilenos, esto contribuyó a fortalecer el desarrollo de las rutas del alambique y el aguardiente.

Los productores chilenos lograron construir una amplia red de distribución de sus alcoholes, llegando a abastecer los mercados de Potosí en el norte y de Buenos Aires por el este. Los grandes espacios geoculturales creados por el Imperio Español facilitaron el surgimiento de estas extensas rutas comerciales, lo que facilitó la especialización de la producción de cobre labrado (sobre todo alambiques), vinos y aguardientes.

En torno al mercado de Potosí, el abastecimiento de aguardiente desencadenó una intensa competencia entre los productores peruanos y chilenos. En los siglos XVII y XVIII la producción chilena fue cuantitativamente inferior a la del Perú; pero fue suficiente para disputar una porción considerable del mercado potosino, ello llevó a los hacendados peruanos a desplegar sus influencias ante la corte para solicitar privilegios y monopolios de acceso al mercado de Potosí. Los productores peruanos tenían ventajas sobre los chilenos debido a las menores 
distancias entre el punto de producción y el mercado potosino, y la cercanía de la capital del virreinato y las posibilidades de influencia en la corte. No obstante ello, los chilenos se apoyaron en sus propias fortalezas, sobre todo la superioridad tecnológica del alambique sobre las falcas peruanas, y el servicio regular de transporte terrestre que aseguraban los arrieros. Sobre estas bases, el aguardiente chileno logró mantener su presencia en los mercados regionales durante todo el periodo colonial. De esta manera se echaron las bases para el desarrollo del proceso que condujo a la delimitación de la Denominación de Origen Pisco en 1931.

\section{Agradecimientos}

El presente artículo ha sido preparado en el marco del Proyecto FONDECYT Regular COD 1130096 sobre "Denominaciones de origen de productos agroalimentarios chilenos", financiado por la Comisión Nacional de Investigación Científica y Tecnológica de Chile (CONICYT).

\section{Literatura Citada}

Assadourian, C. S.

1983. El sistema de la economía colonial, México, Editorial Nueva Imagen. 367 pp.

Bazán, A.R.

1995. Historia del Noroeste Argentino. Buenos Aires, Plus Ultra. 434 pp.

Braudel, F.

1994. Bebidas y excitantes, Madrid, Alianza Editorial. 64 p. Carrera, J.

2011. Algo más que mercachifles. Pulperos y pulperías en la campaña bonaerense, 1770-1820, Rosario, Prohistoria. $232 \mathrm{pp}$.

Cortés, $\mathrm{H}$.

2005. El origen, producción y comercio del pisco chileno, 1546-1931, Universum, 20 (2): 41-81.

De las Cuevas, J.

2003. Historia apasionada del Brandy de Jerez, Sevilla, Ediciones Geribel. 276 pp.

Fernández, $\mathrm{S}$.

2005. La cultura del vino en la construcción social de la premeseta murciana y alicantina: algunos modelos de desarrollo local en torno a la simbología del vino (Jumilla y Yecla, Monóver y Villena). Revista murciana de antropología, 12: 209-234. Forbes, R.

1948. A Short History of the Art of Distillation: From the Beginnings Up to the Death of Cellier Blumenthal, Leiden, E. J. Brill. 405 pp.

Huertas, L.

2004. Historia de la producción de vinos y piscos en el Perú. Universum, 19 (2): 44-61.

Huertas, L.

2012. Cronología de la producción del vino y del pisco (Perú 1548-2010), Lima, Editorial Universitaria. 393 pp.

Lacoste, $\mathrm{P}$.

2005. El tropero y el origen de la burguesía en el Cono Sur. Estudos Iberoamericanos, 31 (2): 177-205.

Lacoste, P.; Jiménez, D.; Castro, A.; Rendón, B.; y Soto, N. 2013. A binational Appellation of Origin: Pisco in Chile and Peru. Chilean journal of agricultural research 73 (4): 424-429.

Lacoste, P.; Jiménez, D.; Cruz, E.; Rendón, B.; y Soto, N.

2014a. Pisco y toponimia: impacto de las rutas del aguardiente en el desarrollo de nombres y lugares geográficos en Chile, Perú y Argentina. Idesia, 32 (3): 31-41.
Lacoste, P; Briones, F.; Jiménez, D. y Rendón, B.

2014b. La Denominación de Origen Pisco en Chile: algunos problemas nacionales e internacionales. Idesia, 32 (2): $47-56$.

López, C.

2005. Con la cruz y con el aguardiente: la empresa vitivinícola jesuita en el San Juan colonial, Universum, 2 (20): 82-107.

Maldonado, J.

1999. La formación del capitalismo en el Marco del Jerez. De la vitivinicultura tradicional a la agroindustria vinatera moderna (siglos XVIII y XIX), Madrid, Huerga \& Fierro Editores. 434 pp.

Marchena, J.

2010. La vida y los hombres de las galeras de España (siglos XVI-XVII), tesis doctoral, Madrid, Universidad Complutense de Madrid. 513 pp.

Mayo, C. (dir)

2000. Pulperos y pulperías de Buenos Aires (1740-1830), Buenos Aires, Biblos. 139 pp.

Pinto, J.

1980. Población del Norte Chico en el siglo XVIII. Crecimiento y distribución de una región minero agrícola de Chile, La Serena, Talleres Gráficos de la Universidad del Norte. $178 \mathrm{pp}$.

Pinto, J.

1992. La familia en una Sociedad del Chile Colonial. Las modalidades alternativas al vínculo matrimonial en el Norte Chico, 1700-1800). En: Norambuena, Carmen y Salinas Meza, René (eds.): Demografía, familia e inmigración en España y América. Santiago, coedición Universidad de Chile, USACH, UCV, UMCE y Embajada de España, 91-116.

Rice, $\mathrm{P}$.

2012. Vintage Moquegua. History, wine and archaeology on a colonial Peruvian periphery, Austin, University of Texas Press. 343 pp.

Romano, $\mathrm{R}$.

2004. Mecanismo y elementos del sistema económico colonial americano. Siglos XVI-XVIII, México D.F., El Colegio de México-Fondo de Cultura Económica. 480 p.

Tándeter, E.; Milletich, V.; Ollier, M. M. y Ruibal, B.

1987. The Market of Potosí at the End of the Eighteenth Century. London, University of London. 47 pp. 